\title{
Selection Effects on Dishonest Behavior
}

\author{
Petr Houdek, Štěpán Bahník, Marek Hudík, Marek Vranka \\ Faculty of Business Administration, University of Economics in Prague, Czech Republic
}

\begin{abstract}
Many experimental studies use random assignment to identify factors influencing dishonesty. However, in real-life, people deliberately choose dishonesty-enabling environments. In two laboratory experiments, we let participants self-select in two tasks, one of which enabled them to cheat. We found that participants low in the honesty-humility were more likely to choose the cheating-enabling task. Furthermore, after choosing it, they cheated even more than when they were randomly assigned to it for the first time. When choosing the cheatingenabling task was costly, the interest in it decreased, but those who chose the task anyway cheated even more. An intervention based on social proof aimed to discourage self-selection into the cheating-enabling environment had the opposite effect. The results suggest that immoral individuals are likely to dominate cheating-enabling environments, where they cheat extensively. Interventions trying to limit the choice of these environments may backfire and lead to the selection of the worst fraudsters.
\end{abstract}

Keywords: cheating, self-selection, laboratory experiment, honesty-humility

\section{Statement of relevance}

Why is dishonest behavior more common in some groups than others? One explanation assumes that people in different groups are similar, and therefore, the differences in dishonesty across groups are explained through the differences in the environment, i.e., opportunities to cheat, material gains from cheating, the level of punishment, or the probability of detection. Without downplaying the importance of the environmental factors, this paper highlights the importance of people's characteristics for explaining the inter-group variability. Specifically, we show that opportunities for dishonest behavior attract individuals with a higher propensity to cheat. This selection effect is even more substantial if entering the cheating-enabling environment is costly. The entry costs not only attract individuals with the highest tendency to cheat but also increases the amount of cheating on the individual level. Our results highlight the importance of including the possibility of self-selection in laboratory experiments for greater generalizability of their findings. 


\section{Introduction}

Given the prevalence and high societal costs of dishonesty, many experimental studies have attempted to identify the factors associated with dishonest behavior (e.g., Gino \& Ariely, 2012; Shalvi, Eldar, \& Bereby-Meyer, 2012). Typically, these studies randomly assign participants into experimental and control groups and measure the effect of the manipulated factor. However, people are usually not randomly assigned to their circumstances in the real world; they oftentimes actively choose them. Dishonest individuals choose environments where cheating is easier or even tolerated (e.g., Hanna \& Wang, 2017). For example, dishonest people are more likely to stay in groups led by unethical leaders (Cialdini et al., 2019). Similarly, advisers with a record of misconduct are more likely to seek employment with deceptive financial advisory firms (Egan, Matvos, \& Seru, 2019).

Given the importance of selection in the real world, experiments with random assignment of participants to cheating-enabling conditions may underestimate the extent of cheating in reallife circumstances (e.g., Gino \& Ariely, 2012). Consequently, applicability of effects obtained in experimental studies to real environments is limited (Houdek, 2019). People in real-world cheating-enabling environments are likely to differ from other people due to selfselection (e.g., Houdek, 2017; Swider et al., 2015). Therefore, any factors influencing cheating that are observed in experiments without taking self-selection into account may not affect people in real-world cheating-enabling environments. Additionally, the neglect of selection in experimental studies of dishonesty may result in overlooking potential benefits of interventions targeting the selection.

Although the importance of the selection and sorting effects in various contexts is recognized in the large body of non-experimental literature (e.g., Bayer \& Ross, 2006; Bless \& Burger, 2016), only a handful of experimental studies considered these effects in relation to cheating and dishonesty. Exceptions include Klimm and Loipersberger (2019), who examined whether liars lie more if their potential victims fail to avoid the environment which enables deception. Another study showed that people who believe that they are perceived as honest adopt a deceptive strategy more frequently, and high fines for deception reinforce this selection (Konrad, Lohse, \& Qari, 2014). Finally, Gino, Krupka, and Weber (2013) found that a situation with the possibility to avoid being audited produces more cheating than situations in which an audit is either imposed by an authority or completely absent.

In this paper, we report the results of two experiments, which allowed participants to choose either an environment where they were able to cheat or where cheating was impossible. Both experiments used a modified mind game in which participants were rewarded for correct predictions of die rolls (Jiang, 2013). There were two versions of the game. In one version, the success was determined solely by chance, and participants could not influence it. In the other version, the participants self-reported whether their prediction was correct and therefore they had an opportunity to cheat.

The first experiment examined how people who select the cheating-enabling version of the game differ from others and what effect the self-selection has on cheating. We sampled 
participants from two distinctly different cultures, Czechia and China, thus allowing for greater generalizability of findings (e.g., Mann et al., 2016). In the second experiment, we explored whether financial and social factors affect the selection: In one treatment, we introduced a fee for selecting the cheating-enabling version of the game. In the other treatment, we provided participants information about how many participants chose each version of the game in a previous study. The design of this intervention was motivated by the literature showing that social proof has been effective in changing behavior in multiple domains (e.g., Benartzi et al., 2017).

We found that individuals with a higher propensity to cheat selected themselves into the environment, which allowed them to cheat. Moreover, participants who chose the cheatingenabling version cheated more than those who were assigned to it at random. In the second experiment, we found that the fee discouraged some individuals from choosing the cheatingenabling environment. However, the fee also resulted in more cheating by those who selected this environment. Surprisingly, participants were more likely to choose the cheating-enabling environment after receiving the information that only a low fraction of participants chose this environment in the past.

\section{Study 1}

\section{Methods}

The materials used in the study, data, analysis scripts, as well as preregistration of the study are available at https://osf.io/cj28d/.

\section{Participants}

Six hundred and twenty-two subjects (315 Czech and 307 Chinese, $67 \%$ female, $M_{d n} n_{\text {age }}=21$ ) participated in the study. Both the Czech and Chinese samples were recruited from university laboratories' subject pools. According to a pre-registered exclusion criterion, 27 participants who did not answer all attention checks correctly were excluded from analyses using individual differences measures.

\section{Design and procedure}

The study was conducted in a laboratory setting in groups of up to 17 or 38 participants in Czechia and China, respectively, who worked individually on workstations separated by dividers. The experiment was administered in English (non-native language for both groups) using a custom-written Python program. All rewards were paid in the local currency; CZK in Czechia (100 CZK = approx. 4.40 USD) and CNY in China (100 CNY = 14.40 USD).

The experiment used a modified mind game (Jiang, 2013). Participants earned 35 CZK or 10 CNY for each correct prediction of whether the outcome of a fair die roll would be odd or even. Participants played three rounds of the game, with ten rolls in each round. They were 
informed beforehand that they would receive a financial reward according to their result in one randomly selected round. Participants' understanding of the rules was checked using a short quiz.

There were two different versions of the game: in the BEFORE version, participants had stated their predictions before the die was rolled, and then they saw the outcome. Therefore, no cheating was possible. In the AFTER version, participants were asked to make a prediction in their mind and remember it. Then a roll was made, and participants were shown the outcome and asked to state whether they had predicted it correctly or not. As the actual prediction was only in participants' minds, they could cheat and misreport even their incorrect predictions as correct.

In the first two rounds, participants played one round of the game in the AFTER and one in the BEFORE version, in random order. The first two rounds introduced participants to both versions of the task, and the number of reported predictions in the AFTER version of the task served as a baseline measure of cheating. For the third round, participants were randomly assigned to a control or experimental group, and they read short descriptions of both versions of the game. The participants in the control group were randomly assigned either the AFTER or BEFORE version. The participants in the experimental group were offered a choice whether they wanted to play the third round in the BEFORE or AFTER version, or whether they wanted to be assigned to one of the versions at random. See Figure 1 for a schema of the study.

After finishing the main part of the experiment, we measured participants' risk preferences using a simplified Holt and Laury (HL) task (Teubner, Adam, \& Niemeyer, 2015) and their social preferences by giving them an option to give a part of their reward to a charity of their choice. ${ }^{1}$ Finally, participants answered socio-demographic questions and filled several questionnaires; namely, 60-items HEXACO scale (Ashton \& Lee, 2009), work deception scale (Gunia \& Levine, 2016), prosocialness scale (Caprara, Steca, Zelli, \& Capanna, 2005), desirable responding scale (BIDR-16; Hart, Ritchie, Hepper, \& Gebauer, 2015), moral agency scale (Black, 2016), measure of moral disengagement (Shu, Gino, \& Bazerman, 2011), and values questionnaire (PVQ-RR; Schwartz, 2017). ${ }^{2}$ Participants were informed before the questionnaires that there would be attention check items in the questionnaires and that they could earn an additional reward of $50 \mathrm{CZK}$ or $15 \mathrm{CNY}$ if they would manage to answer all of them correctly.

At the end of the session, participants answered questions about their perception of the two versions of the dice-rolling task and completed a short debriefing in which they answered open-ended questions about the aims of the different parts of the experiment.

\footnotetext{
${ }^{1}$ Because participants could earn up to $100 \mathrm{CZK}$ or $30 \mathrm{CNY}$ in the HL task and they were informed that experimenters would know only the total amount of reward during its payment at the end of the session, participants should not have been worried that a high reward would suggest dishonest conduct in the dice rolling task.

${ }^{2}$ Descriptive statistics for the measures can be found on https://osf.io/r6bne/.
} 


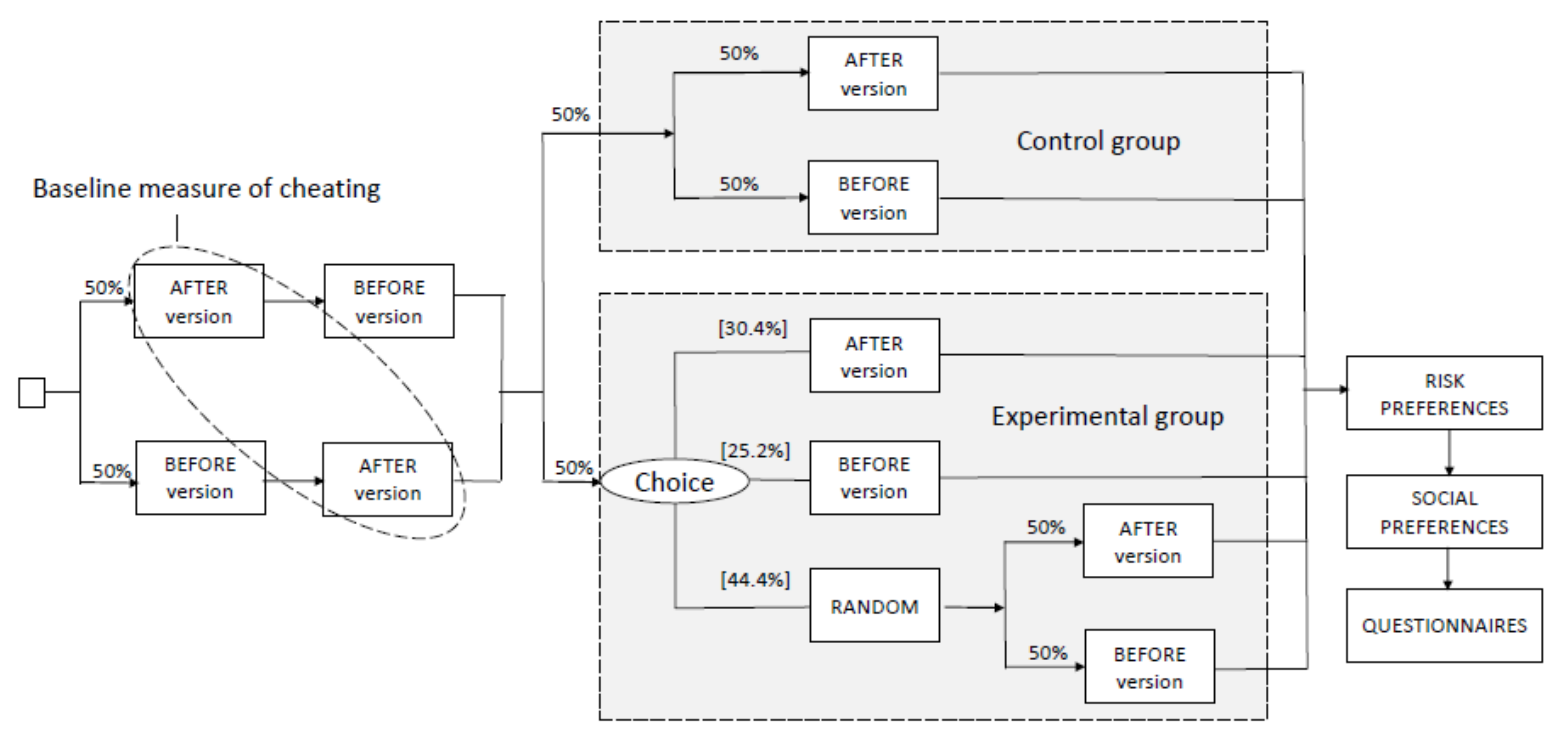

ROUND $1 \quad$ ROUND 2

ROUND 3

Figure 1. Schema of the design of Study 1. In rounds 1 and 2, participants played AFTER and BEFORE versions of the game in a randomized order. The number of reported predictions in the AFTER version of the task served as a baseline measure of cheating. In round 3, participants were randomly selected to a control and experimental group. Participants in the control group were randomly assigned BEFORE or AFTER version of the game. Participants in the experimental group chose whether they wanted to play BEFORE or AFTER version, or whether they wanted to be assigned to one of the versions at random. Then we measured, for all participants, risk and social preferences. Finally, participants answered socio-demographic questions and filled several questionnaires.

\section{Results}

The average number of reported correct predictions in the baseline measure of the AFTER version of the task exceeded five expected by chance, $t(621)=11.25, p<.001, d=0.45,95 \%$ CI $[0.37,0.53], M=5.84$, showing that participants cheated in the task. Czech participants cheated less than Chinese, $t(611)=-7.73, p<.001, d=-0.62,95 \%$ CI $[-0.79,-0.46], M_{\text {Czech }}=$ $5.30, M_{\text {Chinese }}=6.42 .^{3}$

The AFTER version of the task was chosen by $30.4 \%$ of the participants who were given the opportunity to choose a version of the task for the third round and the BEFORE version was chosen by $25.2 \%$ of participants. The remaining participants decided to be assigned the task at random. Participants who chose the AFTER version cheated more than participants in the control group who were assigned the AFTER version randomly, $t(249)=5.01, p<.001, d=$ $0.65,95 \%$ CI $[0.39,0.91], M_{\text {after }}=7.33, M_{\text {control }}=6.07$, as well as more than participants from the experimental group who were assigned the AFTER version randomly, $t(165)=4.93, p<$ $.001, d=0.78,95 \%$ CI $[0.45,1.09], M_{\text {random }}=5.88$. Participants in the experimental group who chose the AFTER version also cheated more in the baseline measure of the AFTER version than those who chose to be assigned randomly, $t(242)=4.02, p<.001, d=0.52,95 \%$

\footnotetext{
${ }^{3}$ Other comparisons of the two samples can be found on https://osf.io/xpbdq/.
} 
CI $[0.26,0.78], M_{\text {after }}=6.56, M_{\text {random }}=5.54$, as well as those who chose the BEFORE version of the task, $t(179)=3.65, p<.001, d=0.54,95 \%$ CI $[0.25,0.84], M_{\text {before }}=5.41$. The latter two groups did not differ significantly from each other, $t(225)=0.53, p=.60, d=0.07,95 \%$ CI $[-0.20,0.34]$. The results therefore showed that cheaters also tended to choose the opportunity to cheat when given a choice.

Participants who chose the AFTER version of the task also cheated more in the third round than in the baseline, $t(98)=4.18, p<.001, d=0.42,95 \%$ CI $[0.21,0.63], M_{\text {after third }}=7.33$, $M_{\text {after baseline }}=6.56$. This shows that participants in the experimental condition who chose the AFTER version did not do so only because they had a high number of correct predictions in the baseline by chance. On the other hand, there was no significant difference in cheating between the baseline and the third round for those in the experimental group who were assigned to the AFTER version randomly, $t(67)=0.35, p=.72, d=0.04,95 \%$ CI $[-0.20$, $0.28], M_{\text {random third }}=5.88, M_{\text {random baseline }}=5.81$, as well as for those in the control group who were assigned to the AFTER version, $t(151)=1.06, p=.29, d=0.09,95 \% \mathrm{CI}[-0.07,0.25]$, $M_{\text {control third }}=6.07, M_{\text {control baseline }}=5.89$. The possibility to choose a version of the task, therefore, increased the rate of cheating for those who wanted to cheat. The experimental group also had somewhat higher earnings in the third round overall than the control group, $t(620)=2.37, p=.02, d=0.19,95 \%$ CI $[0.03,0.35], M_{\text {experimental }}=5.89, M_{\text {control }}=5.53$. This suggests that the possibility of choosing an environment might increase the rate of cheating, at least under certain circumstances.

Out of the included individual differences measures, only honesty-humility predicted both cheating and selection of a version of the task (see Figure 2). Participants higher in honestyhumility cheated less in the baseline measure, $r_{\mathrm{S}}=-.09,95 \% \mathrm{CI}[-.16,-.01], p=.03$, and were also less likely to choose to have an option to cheat in the third round of the task, $r_{\mathrm{S}}=-.13$, $95 \%$ CI [-.24, -.02], $p=.02$.

\section{Reported correct predictions}

Donation to charity (CZE)

Donation to charity ( $\mathrm{CHN})$

Risk aversion

Honesty-Humility

Emotionality

Extraversion

Agreeableness

Conscientiousness

Openness to experience

Desirable responding

Moral agency

Moral disengagement

Prosociality

Work deception $\begin{array}{llllllllllll}0.5 & -0.4 & -0.3 & -0.2 & -0.1 & 0 & 0.1 & 0.2 & 0.3 & 0.4 & 0.5\end{array}$

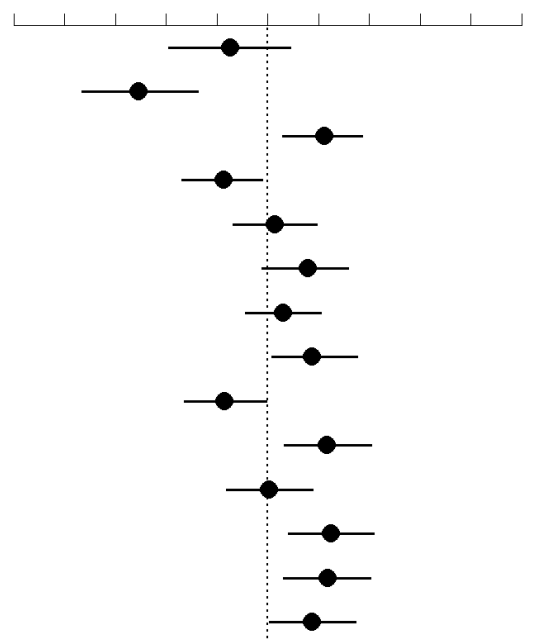

Choice of a version

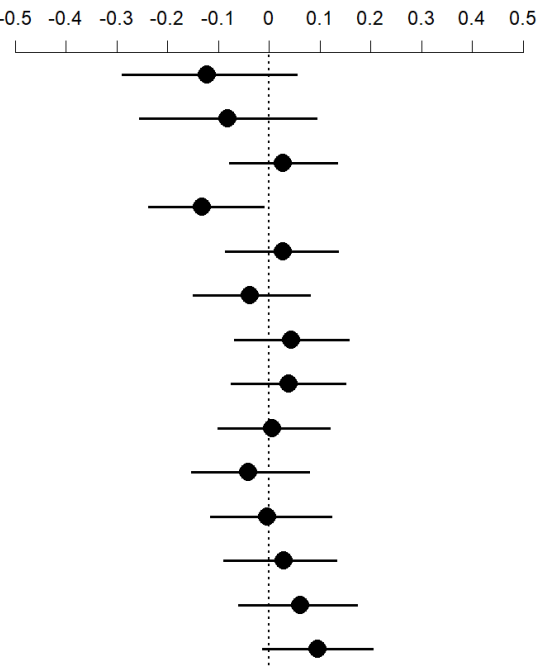

Figure 2. Correlations between individual differences measures with baseline cheating and selection of a version of the task in Study 1. The figure shows Spearman correlation coefficients and $95 \%$ confidence intervals for their estimates. The choice of a version had three ordered levels- 
BEFORE, random, and AFTER - and only participants in the experimental group were included. Donations to charity are analyzed separately for the two samples because the charities, as well as exact monetary rewards differed between them. The results from the PVQ are on https://osf.io/xpbdq/.

\section{Discussion}

The results show that the participants who chose the cheating-enabling version cheated more than those who were assigned to it at random. Furthermore, those who selected the cheating enabling version cheated more than other participants even in the initial rounds before the selection, and they further increased the rate of their cheating after the selection. These findings suggest that current research on dishonesty may underestimate the rate of cheating in the real world, where people can usually choose not only whether to cheat when they have an opportunity, but they might also actively seek this opportunity.

Because cheating was not observable directly, there are alternative explanations of some of our findings. In particular, the positive correlation between the baseline cheating and selection of the AFTER version might have been caused by participants who chose the AFTER version of the game because they were luckier when they first played it. Therefore, they might have believed that the AFTER version is associated with a higher chance of winning than the BEFORE version (Rabin \& Vayanos, 2010). However, this explanation is contradicted by two other results. First, participants who chose the AFTER version for the third round then reported even more correct guesses than in the baseline. Second, low honesty-humility, previously shown to predict cheating (e.g., Kleinlogel, Dietz, \& Antonakis, 2018), was associated both with the high number of correct guesses and with the selection of the cheating-enabling environment. Both results suggest that cheaters, rather than superstitious individuals, selected the cheating-enabling environment.

In Study 1, we assumed zero costs associated with the choice of an environment. However, such entry costs usually exist, and they can have contradictory effects on the prevalence of cheating. On the one hand, entry costs may deter people who are not motivated to cheat. On the other hand, entry costs could lead to more cheating of fraudsters who are prepared to cheat despite the costs, because they might want to recoup them or even view them as a kind of a fee that justifies their subsequent cheating (Shalvi et al., 2015). In Study 2, we tested how entry costs affect the rate of selection and the magnitude of cheating.

We also tested a non-financial measure that could limit the preference of the cheatingenabling environment. A social norm, that is, information about the behavior of others in a given situation, is a powerful signal of how one should behave. A reminder of a social norm usually leads to more congruent behavior. Nevertheless, the evidence about its effectiveness is inconclusive (e.g., Bicchieri \& Dimant, 2019; Schultz et al., 2007). Because a minority of participants chose both the cheating-enabling (30\%) and the cheating-prohibiting environment (25\%) in Study 1, we were able to manipulate perceived norms by informing participants about either of the proportions in Study 2. 


\section{Study 2}

\section{Methods}

The materials used in the study, data, analysis scripts, as well as preregistration of the study are available at https://osf.io/cj28d/.

\section{Participants}

The experiment was conducted with 501 participants $\left(61 \%\right.$ female, $\left.M_{d n_{a g e}}=22\right)$ from our Czech laboratory subject pool consisting mostly of university students $(n=352)$. The most represented fields were humanities and social sciences $(n=125)$ and economics and management $(\mathrm{n}=101)$. According to a pre-registered exclusion criterion, nine participants who did not answer all the attention checks correctly were excluded for analyses using questionnaire data.

\section{Design and procedure}

The study was conducted in a laboratory setting in groups of up to 17 participants, who worked individually on workstations separated by dividers. The present study was the first in a batch of three unrelated studies. The whole experiment was administered in Czech using a custom-written Python program.

Participants played five rounds of the same modified mind game as in Study 1 with twelve rolls in each round. The reward for correctly predicting whether the outcome will be odd or even increased by $5 \mathrm{CZK}$ with each correct prediction from $5 \mathrm{CZK}$ for the first correct prediction to $60 \mathrm{CZK}$ for the twelfth. Participants were informed that they would receive the money earned in one randomly selected round. In order to prevent participants' concerns that a higher reward would be an indicator of dishonest conduct, they were also informed that after the main dice rolling task, there would be a lottery task in which they could earn an additional reward of up to $1280 \mathrm{CZK}$ and that experimenters would know only the total amount of reward during its payment at the end of the session.

As in Study 1, in the first two rounds, every participant played one round of the game in the AFTER and one in the BEFORE version, in random order. Before the third round began, participants had read short descriptions of both versions of the game, and then they chose whether they wanted to play the next round in the BEFORE or AFTER version. One half of the participants had to pay a fee of $25 \mathrm{CZK}$ for the selection of the AFTER version. The other half of the participants could select the AFTER version without any fee. The BEFORE version was not associated with any fee for any of the participants. The fourth round was the same as the third, but participants were given the condition (fee or no-fee) that they had not received in the third round. In the fifth round, participants again chose between the BEFORE and AFTER version, this time without any fees. However, before making their decision, one half of participants learned that "only $30 \%$ " of participants wanted to participate in the 
AFTER version in a similar previous experiment and the other half learned that "only $25 \%$ " of participants wanted to participate in the BEFORE version of the task. ${ }^{4}$ See Figure 3 for a schema of the study.

At the end of the session, participants provided socio-demographic information, filled several questionnaires - namely, 60-items HEXACO scale (Ashton \& Lee, 2009) and Short Dark Triad scale (Jones \& Paulhus, 2014) ${ }^{5}$ - answered questions about their perception of the two versions of the dice-rolling task, and completed a short debriefing in which they answered open-ended questions about the aims of the different parts of the experiment. Attention check items were again added to the questionnaires, and participants could earn an additional reward of $50 \mathrm{CZK}$ if they answered all of them correctly.

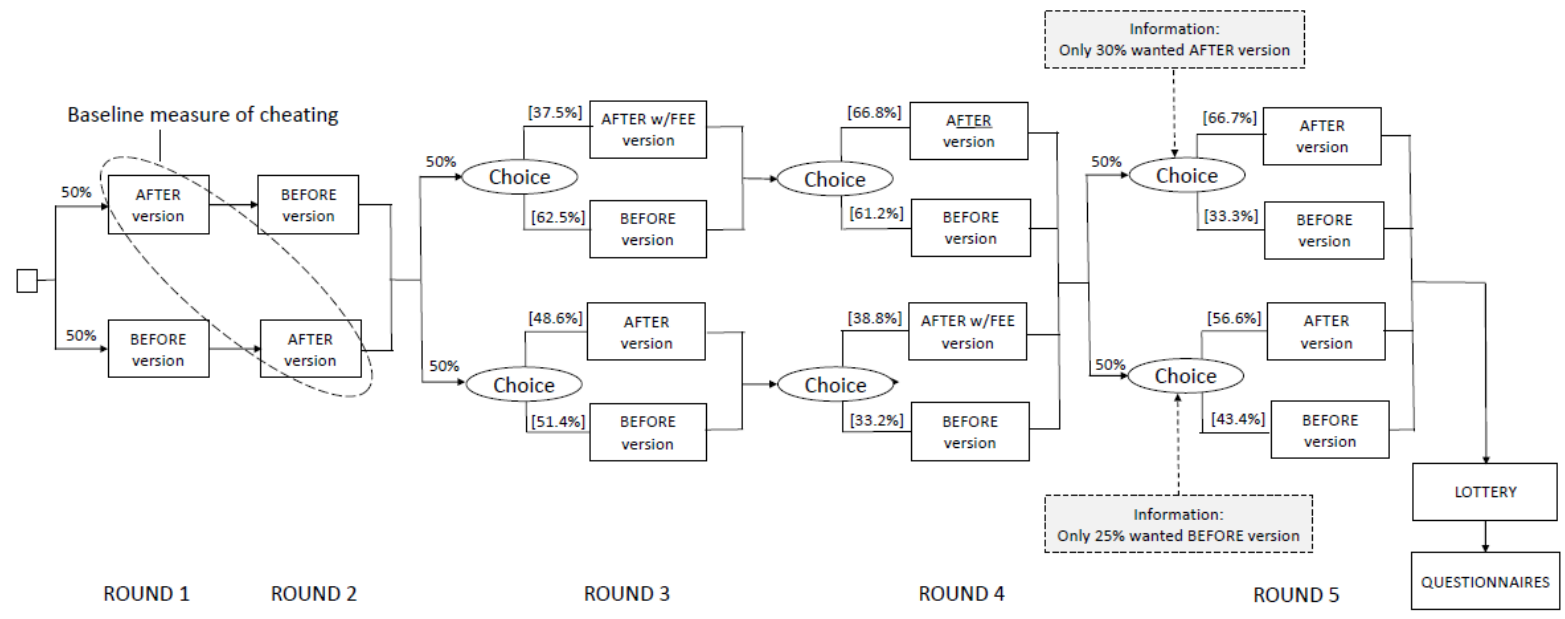

Figure 3. Schema of the design of Study 2. In rounds 1 and 2, participants played AFTER and BEFORE versions of the game in a randomized order. The number of reported predictions in the AFTER version of the task served as a baseline measure of cheating. In rounds 3 and 4 , participants chose in random order between the BEFORE and AFTER version, and between the BEFORE and AFTER version with a fee. In round 5, participants chose between the BEFORE and AFTER version. Before making their decision, a half of participants learned that "only $30 \%$ " of participants participated in the AFTER version in a previous experiment while the other half learned that "only 25 $\%$ " of participants participated in the BEFORE version of the task. Finally, app participants played a lottery, answered socio-demographic questions, and filled several questionnaires.

\section{Results}

The average number of reported correct predictions in the baseline measure of cheating exceeded six expected by chance, $t(500)=17.15, p<.001, d=0.77,95 \%$ CI $[0.67,0.87], M$ $=7.68$, showing that participants cheated in the task. A linear regression with the reported number of correct predictions in the baseline measurement as the dependent variable, the number of selections of the AFTER version of the task in the third and fourth rounds (coded

\footnotetext{
${ }^{4}$ We asked participants about their prediction of how many other participants would choose each of the versions of the task. On average, participants predicted that $28.2 \%$ of participants $(\mathrm{SD}=19.3 \%$ ) would choose the BEFORE version of the task, suggesting that participants should have considered the information about the proportion of participants choosing the AFTER version of the task as low as we intended.

${ }^{5}$ Descriptive statistics for the measures can be found on https://osf.io/r6bne/.
} 
as $-0.5,0$, and 0.5 ), the round with the fee condition, and their interaction as predictors, showed that participants who selected the AFTER version of the task in the third and fourth rounds a higher number of times reported a higher number of correct predictions in the baseline measurement, $t(497)=13.30, p<.001, b=2.88,95 \%$ CI $[2.45,3.30]$.

In the third round of the task, the AFTER version was chosen by $37.5 \%$ of participants in the fee condition and $48.6 \%$ of participants in the no-fee condition. In the fourth round, the AFTER version was chosen by $38.8 \%$ of participants in the fee condition and $66.8 \%$ of participants in the no-fee condition. A mixed-effect logistic regression with the choice of the version as the dependent variable, the presence of a fee, round number and their interaction as predictors, showed that participants were less likely to select the AFTER version in the presence of a fee, $z=-6.49, p<.001, O R=0.34,95 \%$ CI $[0.25,0.48]$, and more likely in the fourth round of the task, $z=3.50, p<.001, O R=1.71,95 \%$ CI $[1.27,2.31]$. The difference between the fee and no-fee condition was larger in the fourth round, $z=-2.49, p=.01$, ratio of $\mathrm{OR}=0.40,95 \% \mathrm{CI}[0.19,0.82]$. When the difference between rounds was analyzed separately for the two conditions, there was no difference between the two rounds for the fee condition, $z=0.29, p=.77, O R=1.06,95 \%$ CI $[0.74,1.51]$, but the AFTER version was more likely to be selected in the fourth round than in the third round in the no-fee condition, $z$ $=4.10, p<.001, O R=2.13,95 \%$ CI $[1.48,3.06]$.

Next, to test the effect of the presence of a fee on cheating, we performed a linear mixedeffect regression with the number of reported correct predictions as the dependent variable and the presence of a fee, round number, and their interaction as predictors for the 144 participants who chose the AFTER version of the task in both third and fourth rounds. The number of reported correct predictions did not differ significantly between the two rounds, $t(142.0)=1.53, p=.13, b=0.15,95 \%$ CI [-0.04, 0.35]. Participants reported more correct predictions in the presence of a fee, $t(142.0)=4.54, p<.001, b=0.45,95 \% \mathrm{CI}[0.26,0.65]$, and this effect did not differ significantly based on the round of the task, $t(142.0)=0.56, p=$ $.58, b=0.36,95 \%$ CI $[-0.92,1.65]$. Participants reported, on average, 10.48 correct predictions in the presence of a fee and 10.05 correct predictions in its absence. The average reward in the presence of the fee was $310.5 \mathrm{CZK}$ and 288.2 CZK in its absence. The increased cheating therefore almost fully compensated the $25 \mathrm{CZK}$ fee. Participants who chose the AFTER version only in the round when there was no fee also reported significantly more correct predictions than the expected six. Such participants who had the no-fee condition in the third round cheated less than the participants who had no fee in the fourth round, $t(144)=-2.92, p=.004, d=-0.49,95 \%$ CI $[-0.83,-0.16], M_{\text {third no-fee }}=6.83, M_{\text {fourth no- }}$ fee $=7.80$.

To test the effect of the information about the share of participants who chose the AFTER or BEFORE version of the task, we conducted a logistic regression with the choice of the version in the fifth round as the dependent variable and the information condition as a predictor. The model included the number of choices of the AFTER version in third and fourth rounds as a covariate. Contrary to our prediction, participants who received the information that a low proportion of participants had chosen the AFTER version were more likely to choose the AFTER version of the task, $t(498)=2.41, p=.02, O R=1.09,95 \% \mathrm{CI}$ 
$[1.02,1.17]$. The AFTER version of the task was chosen by $66.7 \%$ of participants who were informed that a low proportion of participants had chosen the AFTER version, and $56.6 \%$ of participants who were informed that a low proportion had chosen the BEFORE version. The number of reported correct predictions did not significantly differ between the participants who chose the AFTER version in the two conditions, $t(307)=-1.55, p=.12, d=-0.18,95 \%$ CI $[-0.40,0.05], M_{\text {low after }}=8.75, M_{\text {low before }}=9.18$.

Out of the included individual differences measures, honesty-humility, machiavellism, and psychopathy predicted both cheating and selection of a version of the task (see Figure 4). Participants higher in honesty-humility cheated less in the baseline measure, $r_{\mathrm{S}}=-.24,95 \%$ CI $[-.33,-.16], p<.001$, and also chose to have an option to cheat in the third and fourth rounds of the task less frequently, $r_{\mathrm{S}}=-.30,95 \% \mathrm{CI}[-.38,-.22], p<.001$. On the other hand, participants higher in machiavellism were more likely to cheat in the baseline, $r_{\mathrm{S}}=.18,95 \%$ CI $[.09, .27], p<.001$, and chose to have an option to cheat in the third and fourth rounds of the task more frequently, $r_{\mathrm{S}}=.17,95 \% \mathrm{CI}[.06, .26], p<.001$. Similarly, participants higher in machiavellism were more likely to cheat in the baseline, $r_{\mathrm{S}}=.12,95 \% \mathrm{CI}[.04, .21], p=$ .006 , and chose to have an option to cheat in the third and fourth rounds of the task more frequently, $r_{\mathrm{S}}=.11,95 \%$ CI $[.02, .20], p=.02$.

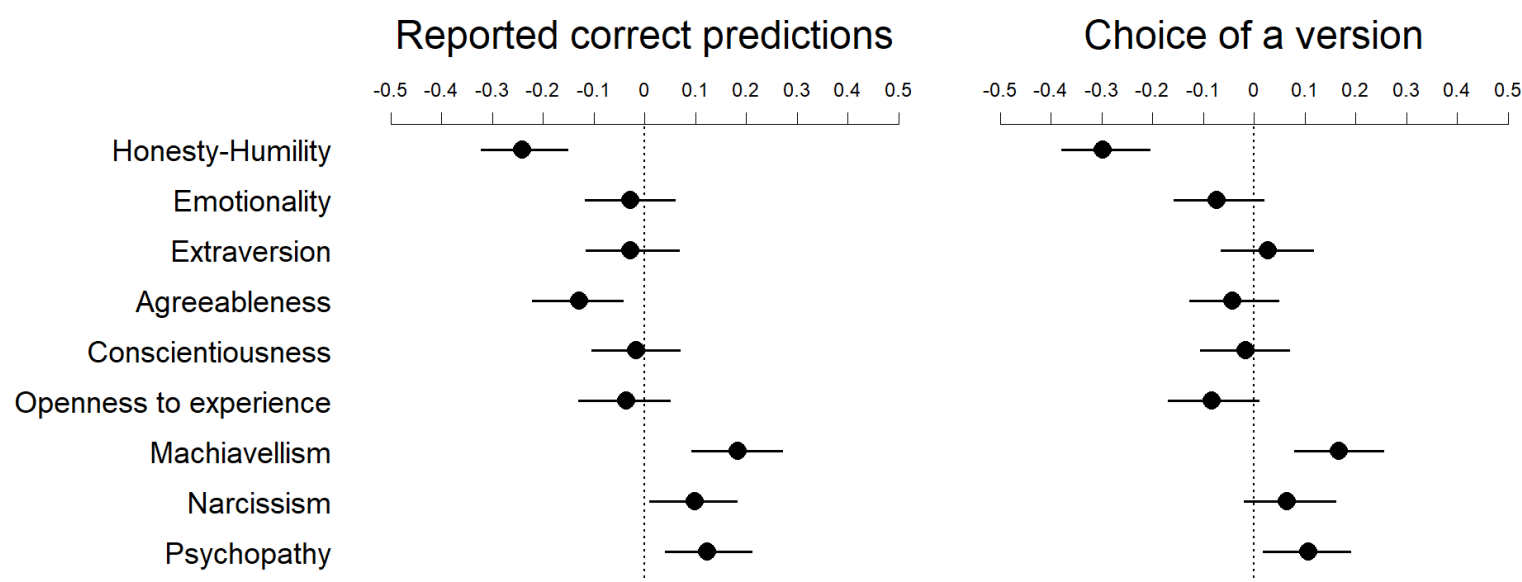

Figure 4. Correlations between individual differences measures with baseline cheating and selection of a version of the task in Study 2. The figure shows Spearman correlation coefficients and $95 \%$ confidence intervals for their estimates. The choice of a version corresponds to the number of choices of the AFTER version in the third and fourth rounds of the task.

\section{Discussion}

The second study replicated the results of Study 1, showing that participants who selected the cheating-enabling environment tended to cheat more. The negative association of honestyhumility with the selection of the cheating-enabling environment was also replicated. Moreover, we observed that participants high in Machiavellism and psychopathy were more likely to choose the cheating-enabling environment. Our findings thus confirm that people who select cheating-enabling environments differ from those who do not. 
Introduction of a fee for entering the cheating-enabling environment decreased the number of participants who selected this environment; however, participants who chose it despite the fee then cheated more. The fact that increasing costs of entering or staying in a cheating-enabling environment may lead to more intense cheating, has important policy implications. Payments in many occupations, such as finance consultant, or real estate agent, at least partly depend on self-reported performance, which enables dishonest behavior. In order to perform these occupations, individuals have to purchase a license or incur other costs. Our findings suggest not only that these types of occupations may attract more dishonest individuals but also that the licensing could result in increased cheating.

The intervention based on social norms that we employed with the goal of reducing the number of participants choosing the cheating-enabling environment did not have the intended effect. The participants who were informed that only a low proportion of participants had selected the cheating-enabling environment were more likely to choose this environment. A possible explanation is that participants thought that with a small number of cheaters, it is justifiable to cheat because not so many resources is going to be misused. This explanation is also supported by the fact that participants themselves estimated much higher proportion of cheaters $(72 \%)$. Nevertheless, our data do not allow us to test these conjectures.

We found that participants chose the AFTER version of the game with no fee more often in the fourth round than in the third round. It is possible that participants' choice in the fourth round was affected by the removal of the fee. In other words, participants who were aware that selection of the AFTER version can be costly perceived the selection in the no-fee treatment as "free" and, therefore, more tempting (Shampanier, Mazar, \& Ariely, 2007). These results are also in line with findings by Khadjavi (2014), who observed an increase in dishonest behavior when the possibility of punishment was removed in a laboratory stealing game. This suggests that if a cheating-enabling environment suddenly becomes easier to access, people may be more willing to choose it not only because of the lack of the additional costs but also because of the favorable comparison to the previous costly state.

\section{General discussion}

We found that people choose or avoid cheating environments based on their personality and preferences. In the light of our results, we recommend enriching the experimental methodology by including the possibility of selection of conditions by participants. Experimental designs typically involve measurement of behavior in assigned conditions, and even participants who would not encounter these conditions in real life are forced to deal with them in an experiment. Inclusion of the possibility of self-selection of participants in different conditions would allow for generalization of experimental findings and it would thus improve external validity of experiments.

From a practical perspective, our results show the importance of influencing self-selection of people into companies, departments, and other groups. If individuals motivated only by selfinterest perceive public office as an opportunity to enrich themselves, people with low moral 
character will seek to become civil servants and politicians. Indeed, several studies confirm this empirically (e.g., Hanna \& Wang, 2017). On the other hand, the self-selection of honest people exists in the Danish public sector (Barfort et al., 2019). Selection of honest people in occupations in which dishonesty may have high societal costs could often be more effective than efforts trying to reduce dishonesty of people who have already chosen them.

The design used in this article can be further extended in various ways. Since reasons for selfselection into groups may vary, self-selection may result in a specific composition of a group, which can further influence behavior of its present or future members. For example, in certain professions (investment banker, salesperson, advertiser), dishonesty or deception could be perceived as a signal of a person's skills, and honest people may therefore avoid them. This could eventually lead to persistent dishonesty in these professions (Gunia \& Levine, 2016). Another possibility for extension is to examine whether selection affects enforcement and punishment. With more cheaters, enforcement and punishment may be more diffused, which may attract additional cheaters in the group (Conley \& Wang, 2006). While we have considered a monetary fee associated with the choice of the cheating-enabling environment, another possibility is to include non-monetary costs of choosing the cheating-enabling environment. Finally, all the cheating behavior in our experiments might have been perceived as victimless. Future research may examine self-selection in cases where cheating has identifiable victims.

\section{Author contributions}

P. Houdek developed the study concept. All authors contributed to the study design. Testing and data collection were performed by M. Vranka and M. Hudík. Š. Bahník performed the data analysis and interpretation. All authors drafted the manuscript. All authors approved the final version of the manuscript for submission.

\section{Funding and Acknowledgments}

We would like to thank Xiyan Cai, Nikola Frollová, Markéta Sýkorová, Dominik Strríbrný, Tony So, Jenny Wang, Yanning Zeng, and Vojtěch Zíka for research assistance. The research was supported by GACR Project No. 18-13766S. The funder had no role in study design, data collection and analysis, or preparation of the manuscript. 


\section{References}

Ashton, M. C., \& Lee, K. (2009). The HEXACO-60: A short measure of the major dimensions of personality. Journal of Personality Assessment, 91(4), 340-345. doi: $10.1080 / 00223890902935878$

Barfort, S., Harmon, N. A., Hjorth, F., \& Olsen, A. L. (2019). Sustaining honesty in public service: The role of selection. American Economic Journal: Economic Policy, 11(4), 96-123.

Bayer, P., \& Ross, S. L. (2006). Identifying individual and group effects in the presence of sorting: A neighborhood effects application (No. w12211). National Bureau of Economic Research.

Benartzi, S., Beshears, J., Milkman, K. L., Sunstein, C. R., Thaler, R. H., Shankar, M., ... \& Galing, S. (2017). Should governments invest more in nudging? Psychological Science, 28(8), 1041-1055.

Bicchieri, C., \& Dimant, E. (2019). Nudging with care: The risks and benefits of social information. Public Choice, Forthcoming.

Black, J. E. (2016). An Introduction to the Moral Agency Scale. Social Psychology, 47, 295310. doi: $10.1027 / 1864-9335 / \mathrm{a} 000284$

Bless, H., \& Burger, A. M. (2016). A Closer Look at Social Psychologists' Silver Bullet. Perspectives on Psychological Science, 11(2), 296-308. doi: 10.1177/1745691615621278

Caprara, G. V., Steca, P., Zelli, A., \& Capanna, C. (2005). A new scale for measuring adults' prosocialness. European Journal of Psychological Assessment, 21(2), 77-89. doi: $10.1027 / 1015-5759.21 .2 .77$

Cialdini, R., Li, Y. J., Samper, A., \& Wellman, N. (2019). How Bad Apples Promote Bad Barrels: Unethical Leader Behavior and the Selective Attrition Effect. Journal of Business Ethics, 1-20.

Conley, J. P., \& Wang, P. (2006). Crime and ethics. Journal of Urban Economics, 60(1), 107-123.

Egan, M., Matvos, G., \& Seru, A. (2019). The market for financial adviser misconduct. Journal of Political Economy, 127(1), 233-295.

Gino, F., \& Ariely, D. (2012). The dark side of creativity: original thinkers can be more dishonest. Journal of Personality and Social Psychology, 102(3), 445-459.

Gino, F., Krupka, E. L., \& Weber, R. A. (2013). License to cheat: Voluntary regulation and ethical behavior. Management Science, 59(10), 2187-2203.

Gunia, B. C., \& Levine, E. E. (2016). Deception as competence: the effect of occupation on ethical judgment and behavior. Academy of Management Proceedings, 2016, 10780. doi: 10.5465/AMBPP.2016.10780abstract

Hanna, R., \& Wang, S. Y. (2017). Dishonesty and selection into public service: Evidence from India. American Economic Journal: Economic Policy, 9(3), 262-90. 
Hart, C. M., Ritchie, T. D., Hepper, E. G., \& Gebauer, J. E. (2015). The balanced inventory of desirable responding short form (BIDR-16). Sage Open, 5(4), doi:

$10.1177 / 2158244015621113$

Houdek, P. (2017). A Perspective on Research on Dishonesty: Limited External Validity Due to the Lack of Possibility of Self-Selection in Experimental Designs. Frontiers in Psychology, 8(1566), 1-6. doi: 10.3389/fpsyg.2017.01566

Houdek, P. (2019). Is Behavioral Ethics Ready for Giving Business and Policy Advice? Journal of Management Inquiry, 28(1), 48-56. doi: 10.1177/1056492617712894

Jiang, T. (2013). Cheating in mind games: The subtlety of rules matters. Journal of Economic Behavior \& Organization, 93, 328-336. doi: 10.1016/j.jebo.2013.04.003

Jones, D. N., \& Paulhus, D. L. (2014). Introducing the short dark triad (SD3) a brief measure of dark personality traits. Assessment, 21(1), 28-41. doi: 10.1177/1073191113514105

Khadjavi, M. (2014). On the interaction of deterrence and emotions. The Journal of Law, Economics, \& Organization, 31(2), 287-319.

Kleinlogel, E. P., Dietz, J., \& Antonakis, J. (2018). Lucky, competent, or just a cheat? Interactive effects of honesty-humility and moral cues on cheating behavior. Personality and Social Psychology Bulletin, 44(2), 158-172.

Klimm, F., \& Loipersberger, F. (2019). Choice as Justification for Dishonesty. Journal of Behavioral and Experimental Economics.

Konrad, K. A., Lohse, T., \& Qari, S. (2014). Deception choice and self-selection - The importance of being earnest. Journal of Economic Behavior \& Organization, 107, 25-39. doi: 10.1016/j.jebo.2014.07.012

Mann, H., Garcia-Rada, X., Hornuf, L., Tafurt, J., \& Ariely, D. (2016). Cut From the Same Cloth: Similarly Dishonest Individuals Across Countries. Journal of Cross-Cultural Psychology, 47(6), 858-874. doi: 10.1177/0022022116648211

Rabin, M., \& Vayanos, D. (2010). The Gambler's and Hot-Hand Fallacies: Theory and Applications. The Review of Economic Studies, 77(2), 730-778. doi: 10.1111/j.1467937X.2009.00582.x

Schultz, P. W., Nolan, J. M., Cialdini, R. B., Goldstein, N. J., \& Griskevicius, V. (2007). The Constructive, Destructive, and Reconstructive Power of Social Norms. Psychological Science, 18(5), 429-434. doi: 10.1111/j.1467-9280.2007.01917.x.

Schwartz, S. H. (2017). The refined theory of basic values. In Values and behavior (pp. 5172). Springer, Cham.

Shalvi, S., Eldar, O., \& Bereby-Meyer, Y. (2012). Honesty Requires Time (and Lack of Justifications). Psychological Science, 23(10), 1264-1270. doi: 10.1177/0956797612443835

Shalvi, S., Gino, F., Barkan, R., \& Ayal, S. (2015). Self-serving justifications: Doing wrong and feeling moral. Current Directions in Psychological Science 24(2): 125-130. doc: $10.1177 / 0963721414553264$ 
Shampanier, K., Mazar, N., \& Ariely, D. (2007). Zero as a special price: The true value of free products. Marketing science, 26(6), 742-757.

Shu, L. L., Gino, F., \& Bazerman, M. H. (2011). Dishonest deed, clear conscience: When cheating leads to moral disengagement and motivated forgetting. Personality and Social Psychology Bulletin, 37(3), 330-349. doi: 10.1177/0146167211398138

Swider, B. W., Zimmerman, R. D., \& Barrick, M. R. (2015). Searching for the right fit: Development of applicant person-organization fit perceptions during the recruitment process. Journal of Applied Psychology, 100(3), 880-893. doi: 10.1037/a0038357

Teubner, T., Adam, M., \& Niemeyer, C. (2015). Measuring risk preferences in field experiments: Proposition of a simplified task. Economics Bulletin, 35(3), 1510-1517. 